\title{
Elite agraria y Estado: influencia de los intereses de los terratenientes en el aparato estatal (184,0-1860)
}

\author{
Agrarian elite and State: influence of the thought \\ of the landowners in the state apparatus (1840-1860) \\ Ariel Rojas Bascuñan \\ Pedagogía en Historia y Ciencias Sociales \\ Universidad de Valparaíso-CHILE \\ arielrojas156@gmail.com
}

\section{Resumen}

El presente artículo realiza un análisis cualitativo de la relación entre la elite agraria y el Estado chileno a mediados del siglo XIX, examinando cómo influyen los intereses de dicho sector en el aparato estatal. El estudio se perfila desde las consecuencias del ciclo triguero californiano (1849-1855), proceso que afecta directamente los intereses de los terratenientes. Para ello, utilizamos como fuente principal las dos primeras revistas de la Sociedad Nacional de Agricultura, institución que se caracteriza por agrupar un importante número de la oligarquía nacional. Así, podemos constatar que la elite agraria comprende al Estado como una institución cuyo principal objetivo es proteger y fomentar la agricultura de los grandes propietarios; mientras que, desde el Estado, existen respuestas positivas ante las presiones y necesidades del sector agrario, principalmente durante el periodo de prosperidad originado por la fiebre del oro en California y Australia.

Palabras claves: Elite agraria, SNA, Estado, siglo XIX.

\begin{abstract}
This article carries out a qualitative analysis of the relationship between the agrarian elite and the Chilean State in the mid-19th century, examining how the approaches of this sector influence the State. The study is outlined from the consequences of the first wheat cycle (1849-1855), a process that directly affects the interests of the landowners. For this reason, we use as our main source the first two journals of the National Agricultural Society, an institution that is characterized by bringing together an important number of the national oligarchy. Thus, we can see that agrarian elite understands the State as an institution whose main objective is to protect and promote the agriculture of large landowners; while the State, there are positive responses to the pressures and needs of the agricultural sector, especially during the period of prosperity caused by the gold rush in California and Australia.
\end{abstract}

Keywords: Agrarian elite, NAS, State, nineteenth century. 
Recibido: 08 de julio de 2020 - Aceptado: 15 de octubre de 2020

\section{Introducción}

En comparación con los demás países de América Latina, después de la independencia, Chile pudo iniciar una fase institucional y de organización nacional rápidamente. El predominio de una economía agropecuaria, el reducido tamaño del territorio y una elite cohesionada que se concentraba en Santiago, no permitió que se desarrollaran grandes conflictos caudillistas a causa de las diferencias regionales. La Batalla de Lircay, en 1830, permitió a un sector de la oligarquía tomar el poder, legitimarse y crear una estructura estatal que les posibilitara gobernar de forma efectiva. Este sector estaba compuesto, principalmente, por grandes terratenientes.

Nuestra investigación aborda la relación entre la elite agraria y el Estado, analizando la influencia de sus intereses en el aparato estatal entre los años 1840 y 1860 . En cuanto a la utilización del término "elite", Antonio Acosta (2014) aludiendo a su definición original y teórica, menciona que su uso solamente debiese aplicarse a grupos dominantes que son elegidos por naturaleza. Por este motivo, para el caso latinoamericano, durante el siglo XIX, sería improcedente, ya que las personas que disponen del poder político y económico lo obtuvieron por medio de procesos históricos que les permitieron apropiarse de los recursos naturales, mano de obra y restringir los espacios políticos. No obstante, como menciona Joingnant (2009), desde principios del siglo XX, principalmente con la escuela elitista italiana (con Geaneto Mosca y Wilfredo Parapeto como los exponentes más destacados), el concepto ha tomado un significado más general, analizando las relaciones sociales y de poder entre grupos gobernantes y gobernados.

Para especificar el uso de la palabra "elite", utilizaremos una cita de Rafael Sagredo (1996) en su estudio sobre la historiografía de las elites chilenas: "el concepto elite entendemos asociado a una minoría selecta con ascendiente sobre el resto de la sociedad en el área que se desempeña como tal. Esto implica que en cada sociedad no existe una única elite que abarque todos los campos de acción existentes en su interior" (104). De acuerdo con el autor, es más apropiado hablar de elites que de elite, ya que dentro de una sociedad existen varios tipos de elite. A pesar de ello, el término "elite" es utilizado, incluso por Sagredo, para referirse al conjunto de grupos (económicos, sociales y políticos) que tenían posiciones dominio o privilegios en la sociedad chilena. Es por ello, que dentro de esos grupos dominantes podemos encontrar una elite agraria.

La condición de elite en Chile durante el siglo XIX se relaciona directamente con la posesión de grandes haciendas. Según Marcelo Carmagnani (1984) la importancia de las haciendas no se fundamenta únicamente en los recursos económicos que éstas otorgaban, sino que responde a algo mucho más profundo. La hacienda sería el elemento básico desde el que se organiza el poder social de la oligarquía. Así mismo, la hacienda 
entrega un prestigio que las demás actividades económicas (comercio, banca, minería) nunca concederán. En consecuencia, el prestigio y los privilegios que otorga poseer una hacienda les permite a los terratenientes posicionarse en los principales puestos políticos del país. Arnold Bauer (1975), analizando la participación política de los terratenientes a lo largo del siglo XIX y principios del XX, afirma que incluso a pesar de que hubo periodos de acelerado crecimiento económico en los sectores no agrarios, la presencia "terratenientes tradicionales" siempre fue significativa. De acuerdo con los datos de Bauer, el porcentaje de congresales que directamente poseen una gran hacienda es para 1854 un 41\%; en 1874 un 50\%; en 1902 un 57\%; y en 1918 un 48\%. Por lo tanto, el concepto "elite agraria" 1 nos permite correlacionar la dimensión del poder político y económico que tenían los terratenientes.

Desde la década de 1820 y 1830 la minería y el comercio tienen un gran impulso debido al descubrimiento y explotación de minas de plata, cobre y carbón. Minerales que comenzaron a tener una creciente demanda por los países que lideraban la revolución industrial. El desarrollo de estas actividades dio "lugar a la formación de nuevos grupos sociales económicamente poderosos que irrumpen en la escena nacional durante el siglo XIX" (Nazer, 1994: 49). Sin embargo, estos nuevos grupos invierten rápidamente sus fortunas en haciendas y en actividades agroexportadoras. Para el año 1874, dentro de los propietarios con mayores ingresos se encuentras varios nombres que iniciaron sus fortunas con actividades mineras y/o comerciales: Francisco Ignacio Ossa, Blas Ossa, Matías Cousiño, Candelaria G. de Gallo,
Alejando N. de Ossa, Guillermo Watkins, Manuel Gallo, Agustín Edwards, Mariana Brown de Ossa, Ramón Subercaseaux, Joaquín Subercaseaux, Vicente Subercaseaux y Eugenio Matta Vargas (Mattelart et al., 1970). De esta manera, lo fundamental para nuestra investigación es que la agricultura exportadora se vincula con los intereses de la mayor parte de la elite chilena.

Cabe señalar que no consideraremos todas las esferas del Estado, sino que analizaremos al gobierno central (presidencia y ministros) y ambas cámaras del parlamento. Ello, debido a que la investigación se centrará en la promulgación de dos leyes significativas para el sector agrario durante el gobierno de Manuel Montt. Así, las esferas estatales a nivel local (municipalidades, intendencias, gobernaciones, etc.) no inciden directamente en nuestra investigación.

La problemática será analizada desde un proceso coyuntural que inició en 1849: el ciclo triguero originado por las grandes demandas de California, y luego en 1854, por Australia ${ }^{2}$. Esto generó un considerable aumento en la demanda y los precios del trigo, provocando un fomento de la economía agrícola, favoreciendo directamente los intereses de los terratenientes. Por lo tanto, nos centraremos en las consecuencias cualitativas del ciclo triguero californiano, analizando cómo influyeron los intereses de la elite agraria en el Estado en este periodo de prosperidad. Las demandas del ciclo triguero ocurrieron mayormente bajo el decenio del presidente Manuel Montt; por lo tanto, examinaremos su gobierno, conocido por su gran cantidad de obras y medidas modernizantes, que según nuestra lectura, no es algo azaroso ni 
fortuito, sino que responde a un momento histórico donde los grandes propietarios tuvieron la oportunidad para consolidar su poder, dirigiendo una serie de medidas a nivel estatal.

En concreto, la problemática que trabajaremos es cómo el Estado chileno, a mediados del siglo XIX, estuvo sujeto al peso político de la elite agraria. En consecuencia, nos planteamos la siguiente hipótesis: las medidas gubernamentales de la década de 1850 estarían fuertemente influenciadas por la coyuntura de California y Australia, debido a que ambas demandas beneficiaron directamente los intereses de los hacendados, generando un estímulo para crear leyes que favorecieran al sector agrícola exportador. Mientras que nuestro objetivo general será: constatar y analizar la influencia de los intereses de la elite agraria en las políticas implementadas por el Estado a mediados del siglo XIX.

Nuestra fuente principal corresponde a las dos primeras revistas de la Sociedad Nacional de Agricultura. La primera revista de la Sociedad inició en 1838 con el nombre de El Agricultor, que perduró hasta 1849 , cuando se cerró debido a que la "institución entra en un periodo de decadencia" (Izquierdo, 1968: 26). Luego reaparece la SNA en 1856, con una revista llamada El Mensajero de la Agricultura, pero este nuevo periodo solo duró dos años.

Tanto El Agricultor como El Mensajero tuvieron entre sus socios a las personas más sobresalientes de la oligarquía chilena. En rasgos generales, la composición de ambas revistas no varía demasiado; la presencia de intelectuales, hacendados y políticos fueron parte de estos formatos. La primera presidencia de El Agricultor estuvo a cargo de Domingo Eyzaguirre, quien tuvo una destacada participación política y también desempeñó actividades agrícolas. Comenzó como ensayador de la Casa de La Moneda en 1794, también en 1802 dirigió obras de canalización en Maipo. Luego de la independencia fue diputado en 8 ocasiones y gobernador del departamento de Victoria (actual San Bernardo). El Secretario General de la Sociedad fue Antonio García Reyes, abogado y constante participante de la política nacional. En 1836 participó en el Ministerio de Relaciones Exteriores, siendo secretario de Mariano Egaña; fue diputado en 5 ocasiones y en 1849 fue ministro de Hacienda. Dentro de la lista de socios, podemos encontrar 6 propietarios que se encuentran entre los 11 hacendados con mayores ingresos anuales para los años 1837-1838: Francisco Ruiz Tagle, Juan de Dios Correa, Valentín Valdivieso, Rafael Larraín Moxó, Santiago Larraín Moxó y José Miguel Irarrázaval Alcalde (Llorca-Jaña et al., 2017). Entre estos, todos, excepto Santiago Larraín, fueron diputadosy/o senadores. También fueron socios ilustres intelectuales extranjeros radicados en el país, como Andrés Bello y Claudio Gay. Además, los presidentes de la República José Joaquín Prieto y Manuel Bulnes fueron patronos de la Sociedad. Durante la presidencia de Prieto, sus ministros fueron socios protectores; mientras que al iniciar la presidencia de Manuel Bulnes los socios protectores se renovaron por: Bernardo O'Higgins, Domingo Eyzaguirre, José de San Martín, el obispo Ignacio Cienfuegos y José Joaquín Prieto. 
El Mensajero sigue esta misma línea, su presidente fue Jerónimo Urmeneta, doctor en leyes desde 1834. Fue elegido diputado en 7 ocasiones y una vez senador, también fue presidente de la cámara de diputado 2 veces. Durante los años 1850 y 1856 fue ministro de Hacienda, luego en 1857 fue nombrado ministro del Interior y Relaciones Exteriores hasta 1858. Es importante mencionar que fue hermano de José Tomás Urmeneta, empresario que inició su fortuna con la mina de cobre Tamaya, pero luego diversificaría sus inversiones a la agricultura (tuvo una lucrativa hacienda en Limache), fundiciones, compañía de gas, seguros, préstamos, entre otras actividades. El Secretario General en este periodo de la Sociedad fue Benjamín Vicuña Mackenna. Hijo de Pedro Feliz Vicuña, fundador de El Mercurio de Valparaíso, en 1827. Benjamín, estudió leyes en la Universidad de Chile. Tuvo una carrera política de oposición a los Gobiernos de Bulnes y Montt. A los 19 años fue secretario de la Sociedad de la Igualdad, la cual fue disuelta por Bulnes. Un año después fue exiliado del país debido a su participación en el Motín de Urriola y los alzamientos de Illapel y Aconcagua. En 1853 regresó a Chile para continuar en la política nacional: a contar de 1864 fue diputado en 4 ocasiones y una vez senador, además tuvo una distinguida participación como Intendente de Santiago (1872-1875). El Mensajero, al igual que El Agricultor, tuvo la participación de hacendados con grandes fortunas: de los 48 propietarios con mayores ingresos para el año 1852, 16 fueron parte de este segundo periodo de la SNA (Lloca-Jaña et al., 2017). Otros socios importantes fueron cuatro ministros del presidente Manuel Montt: Francisco Javier Ovalle, Matías Ovalle, Silvestre Ochagavía, y Jerónimo
Urmeneta; también tres futuros presidentes de la República: Domingo Santa María, Aníbal Pinto y Federico Errázuriz.

En cuanto a la composición de la SNA es importante mencionar que no todos los socios eran hacendados, durante el periodo de 1838 a 1849 hubo un $37 \%$ de personas que desempeñaban actividades agrícolas. Respecto a este punto, Bengoa (2015) realizó una observación fundamental: si bien la composición de la Sociedad Nacional de Agricultura no es en su mayoría de agricultores, en Chile la oligarquía no se puede distinguir entre rural y urbana (el primero vinculado al agro y el segundo a las actividades de la clase burguesa), ya que existía un entrelazamiento social de la clase dominante que se puede caracterizar como urbano-rural. A causa de ello, este autor argumenta que la SNA fue una instancia plutocrática y de carácter corporativa. Por otro lado, los socios de la SNA fueron hacendados "progresistas"3, es decir, la fuente no representa el pensamiento de todos los terratenientes del país, sino a quienes tenían más interés en participar de un adelantamiento de la agricultura. Según Claudio Robles (2003) los hacendados progresistas chilenos del siglo XIX se caracterizaban por introducir maquinaria agrícola, diversificar sus plantaciones (principalmente viñas) y crear o mejorar los sistemas de regadíos. Descripción que concuerda con las publicaciones de El Agricultor y El Mensajero.

Gonzalo Izquierdo (1968) afirma que "la relación entre ésta (SNA) y el Gobierno de la República fue de mutuo apoyo", ya que la Sociedad se presenta como un grupo de particulares que persigue el desarrollo económico y social del país. Por su 
parte, el Estado ayuda a la agrupación "buscando en ella una entidad que lo asesore y que actúe, en relación a cuestiones específicas, como intermediaria entre él y los particulares" (Izquierdo, 1968: 39). El Estado le facilitó locales para sus reuniones y una biblioteca, también aportó con funcionarios para las distintas labores que realizaba la Sociedad, y, sobre todo, financió las publicaciones de las revistas, sueldos para algunos empleados y presupuestos de algunas secciones, lo que se mantuvo hasta la creación del Ministerio de Agricultura en 1924. Mientras tanto la Sociedad desarrolló proyectos de carácter público. Además, estaban en constante comunicación respecto de los avances y problemas técnicos que había en el país. Para graficar las conexiones entre el Estado, los hacendados y la SNA podemos destacar que durante el siglo XIX el $24,3 \%$ de los integrantes de la Sociedad fueron miembros del parlamento (Bengoa, 2015: 178) y de los quince propietarios con mayores ingresos del año 1838, siete eran parte de la Sociedad (Llorca-Jaña et al., 2017).

Las fuentes secundarias que utilizaremos son el Manifiesto del Partido Conservador del año 1851, complementado con información sobre lo que pensaba el gobierno de Manuel Montt; y las Sesiones del Congreso Nacional entre los años 1851 y 1856, sobre los debates de la creación de la Caja de Crédito Hipotecario (1855) y la conversión del diezmo en otro impuesto (1854).

Durante la primera mitad de la década de 1850 , se crearon importantes leyes que beneficiaron a la elite agraria: en 1852 se abolieron los mayorazgos, permitiendo que las tierras pudieran entrar al mercado; en 1853 se creó la Provincia de
Arauco, anticipando las acciones que se tomarán en las décadas posteriores; en 1854 se eliminó el impuesto del diezmo y se legislaron las Sociedades Anónimas; en 1855 se creó la Caja de Crédito Hipotecario y en 1856 se promulgó el Código Civil. De esta manera, para ser más concisos en la investigación, nos enfocaremos en dos importantes ámbitos: crédito e impuestos.

La metodología consistirá en realizar un análisis de los planteamientos de El Agricultor (en cuanto a crédito e impuestos), verificando las ideas de la elite agraria previo al ciclo triguero californiano. Luego, por medio de las sesiones legislativas sobre la creación de la Caja de Crédito Hipotecario y la conversión del diezmo, analizaremos cómo se ejecutaron dichas políticas y cómo es su tramitación en el parlamento. Por último, por medio de El Mensajero, examinaremos cuál era la opinión de la elite agraria sobre la aplicación de las políticas mencionadas anteriormente. Paralelamente, con el objetivo de evidenciar la influencia del sector agrario en el Estado, expondremos la participación de actores que se vincularon directamente con la agricultura, la política (principalmente parlamentarios y ministros), y que además fueron socios de la SNA. De esta manera, corroboraremos cómo, por medio de actores que expresan los intereses del sector agrario en los principales espacios de deliberación política nacional, la elite agraria logró concretar políticas que los beneficiaban categóricamente.

El segundo apartado del artículo contextualizará nuestra investigación, es decir, apunta a caracterizar la situación de la agricultura a mediados de siglo XIX y el ciclo triguero californiano. El 
tercer apartado abordará el objetivo general, para ello, nos adentraremos en la información que nos entrega la fuente principal para desarrollar un análisis respecto a creación de la Caja de Crédito Hipotecario y reemplazo del diezmo. De esta manera, podremos a nalizar la influencia de los intereses de la elite agraria en el Estado previo a 1860 .

\section{2) Agricultura chilena a mediados del siglo XIX y el ciclo triguero californiano}

Chile, a mediados del siglo XIX, constituía una sociedad rural con una economía agraria. La minería otorgaba gran cantidad de ingresos a la elite nortina y al Estado, pero la estructura del país era fundamentalmente rural.

Antes de las demandas de California, es decir, antes de 1849, el principal mercado para los productos agrícolas (especialmente trigo) fue Perú. Desde el periodo colonial ya existía una exportación constante de trigo desde Chile hacia Lima. Según los datos de Sergio Sepúlveda (1959), a principios del siglo XVIII se exportaban 100.000 qq.mm. de trigo, cifra no menor teniendo en cuenta que para el año 1847 la cantidad fue de 120.651 qq.mm (Sepúlveda, 1959: 21). Por lo que se puede concluir que existió un constante mercado entre estos países.

Las tierras del Valle Central estaban monopolizadas por terratenientes. Los grandes dueños de la propiedad agrícola concentraban gran parte de los recursos naturales existentes en esta zona. Desde la colonia, el territorio ya estaba constituido; en otras palabras, los límites entre las diferentes propiedades estaban claramente definidos: "las propiedades eran muy extensas, y a fines del siglo (XVIII) ya no había tierras que repartir en mercedes" (Bengoa, 2015: 116). De acuerdo con los datos de Llorca-Jaña et al. (2017), el porcentaje de familias rurales que no poseía tierras para $1832-1834$ era de un $86 \%$. Con las demandas de California y Australia (y más delante, de Inglaterra), los terratenientes comenzaron un proceso de expansión de la agricultura, que consistía en ampliar el área agrícola dentro de sus propiedades, es decir, aumentar el área cultivada para generar una mejor administración. Esto provocó que, desde mediados del siglo XIX, el número de propiedades medianas creciera. De acuerdo con lo anterior, Robles (2003) concluye que, a principios del siglo XX, la estructura agraria se había transformado, dejando atrás la "dicotomía latifundio/minifundio", desarrollándose un sector de propiedades medianas. No obstante, ello no significó una mejor distribución de tierras para los campesinos. Siguiendo lo planteado por Robles (2003), la expansión agrícola de los terratenientes significó la monopolización de los cultivos tradicionales y las nuevas líneas de producción (viticultura y fruticultura). Al mismo tiempo que precarizaban las condiciones de los inquilinos y aumentaba la incorporación de trabajadores asalariados.

El ciclo triguero fue dado por la explosiva e inesperada demanda de trigo por parte de California y Australia. El origen de la demanda en ambos casos es el mismo: el descubrimiento de oro. La población en California y Australia se enfocó en la extracción de dicho metal, por lo que debieron importar los productos alimenticios, principalmente, 
el trigo. El periodo de demanda californiano va desde 1849 a 1853; mientras que el australiano desde 1854 a 1856. Lo efímero de cada periodo es evidente, pero lo que nos interesa es la intensidad y la oportunidad que presentó para los grandes propietarios. Las consecuencias para el mercado del trigo chileno fueron muy considerables, ya que la demanda total se triplicó. Para ser más precisos sobre estos datos, compararemos la cantidad de trigo y harina exportada hacia Perú con respecto a la totalidad de los exportados hacia el Pacífico (incluyendo a Perú), entre 1849 y 1856.

Tabla 1. Exportaciones en qq.mm. a Perú y hacia el Pacífico.

\begin{tabular}{|l|l|l|}
\hline $\begin{array}{l}\text { Años } \\
\text { o }\end{array}$ & Perú & Total hacia el Pacífico \\
\hline 1849 & 128.835 & 227.268 \\
\hline 1850 & 123.679 & 452.488 \\
\hline 11852 & 136.399 & 325.855 \\
\hline 11854 & 93.393 & 305.048 \\
\hline 11856 & & 293.019 \\
\hline
\end{tabular}

Fuente: Sepúlveda (1959: 44 y 49). Cuadros 5 y 8.

Mientras que los precios del trigo y la harina también se elevaron. De acuerdo a los datos de Claudio Véliz (1961) el precio del trigo en 1849 era de 2,21; en 1852 5,04; y en 1854 4,89. Mientras que, para la harina, en 1849 era de 6,27; en 1852 8,43; y en 18548,58 .

El fin del ciclo triguero se originó por la pronta creación de un moderno sector agrícola en
California, dedicado principalmentea la producción de trigo y harina. California cuenta con un clima similar al chileno, por lo cual, los agricultores californianos realizaron grandes inversiones en sus campos, modernizando la producción del cereal. Ya en 1855 California no necesitaba importar alimentos, la producción local satisfacía la demanda de la región; por lo que "a partir de 1856 Chile enfrentó una dura competencia por parte de los nuevos productores, para surtir el Pacífico, e incluso se llegó a ofrecer en Valparaíso la harina californiana" (Bauer, 1975: 87).

Siguiendo las conclusiones de Sergio Sepúlveda (1959) respecto del ciclo triguero, este afirma que California y Australia no tienen la exagerada importancia que tradicionalmente se les han otorgado, ya que estas demandas solo fueron un apoyo para el proceso de expansión agrícola que ya existía. De modo que las consecuencias cuantitativas no tuvieron mayor relevancia, porque si bien se elevaron las exportaciones y los precios, solo fue por unos pocos años. Cualitativamente, según el mismo autor, entonaron la actividad económica y apresuraron el proceso de expansión de la productividad agraria. Desde nuestro punto de vista, las conclusiones de Sepúlveda son acertadas. Sin embargo, las demandas de California y Australia, pese a ser efímeras, fortuitas y poco estables, fueron la primera gran demanda vivida en Chile independiente, ya que no se había exportado trigo en tales cantidades hacia un país fuera de Sudamérica. Además, el incremento de la demanda y precios, aunque fuera por unos pocos años, fue considerablemente alto. En definitiva, para efectos de nuestra investigación, es que el ciclo triguero californiano fue un gran estímulo para 
los terratenientes, quienes al iniciar las demanda no estaban conscientes de que serían poco duraderos, solo algunos más precavidos anunciaron que la época de prosperidad sería limitada.

\section{3) Influencia de los intereses de la elite agraria en el Estado}

En el presente apartado examinaremos la influencia de los intereses de la elite agraria en el Estado, desde una perspectiva cualitativa. Para ello, analizaremos sus posturas e ideas respecto a dos ámbitos sumamente relevantes para los terratenientes: la creación de un mecanismo de crédito agrícola y la eliminación del diezmo. Para tal objetivo, estudiaremos los planteamientos de la elite agraria respecto a estos dos ámbitos, previo al ciclo triguero, a través de El Agricultor. Luego, analizaremos cómo se desarrolló la aprobación de la creación de la Caja de Crédito Hipotecario (1855) y la conversión del diezmo (1854) en el parlamento. Por último, por medio de El Mensajero, examinaremos las opiniones de la elite agraria posterior a la aprobación de dichas políticas. Además, destacaremos la participación de actores que se vinculan directamente con el sector agrario y tienen importantes intervenciones en el proceso legislativo.

\subsection{Impuestos agrícolas}

Durante la primera mitad del sigloXIX, podemos destacar una serie de medidas económicas destinadas proteger y fomentar la agricultura. En ese sentido, en 1834 el gobierno creó una legislación aduanera para establecer una escala de impuestos diferenciales a las importaciones (artículos libres, $5 \%, 10 \%, 15 \%, 30 \%$ y $35 \%)$. En dicha escala, los productos derivados de la agricultura se encuentran en un $30 \%$, es decir, estaban cargados de un alto impuesto, cuyo objetivo era proteger a los productores agrícolas nacionales. En 1851, también durante el gobierno de Manuel Bulnes, se promulgó una nueva Ley de Aduanas, en la cual "respecto a los impuestos de exportación, los productos a de agricultura, la pesca y manufacturas chilenas quedaron libres de pago" (Cavieres, 1988: 121). De esta manera, las políticas económicas tendieron a favorecer a la agricultura.

La Sociedad Nacional de Agricultura, fue una institución desde donde se criticó férreamente la carga de impuestos que tenía la agricultura. En agosto de 1839, se publicó un artículo en El Agricultor, que fue remitido al Gobierno, titulado "Legislación agrícola en Chile", el que hizo una fuerte crítica a los impuestos que gravaban a los productos agrícolas: "No hay un país en mundo sin exceptuar los Estados Berberiscos, cuyos frutos agrícolas estén más estúpidamente cargados de contribuciones fiscales como entre nosotros" (El Agricultor, $\mathrm{N}^{\circ} 6$, agosto de 1839: 2).

Considerando todas las contribuciones que debían pagar los agricultores al momento de exportar, el autor enumera: un $10 \%$ el diezmo, $1 \%$ el catastro, $3 \%$ derecho de caminos, $6 \%$ derechos de exportación, y continúa preguntándose "¿Y en qué país del mundo medrará jamás la agricultura, donde el Fisco se apropia de una quinta parte de lo que se exporta?". Entre todos los porcentajes, el diezmo ocupa el porcentaje más importante, por ello, dicho impuesto es el más criticado en el artículo: "sin ella, nada habría que objetar al expresado 
reglamento”. Dos años después, Antonio García Reyes, critica nuevamente los impuestos a los productos agrícolas: "Bien sabéis que las contribuciones que gravan a la agricultura, obran de unos tiempos remotos en que no se consultaban los principios luminosos de la ciencia que hoy han hecho reguladora de este ramo dela administración pública; otros creadas para obtener recursos en medio de los apuros del Gobierno, se conservan a despecho de los intereses industriales y de los reclamos de la filosofía" (El Agricultor, $\mathrm{N}^{\circ} 20$, diciembre de 1841: 6).

La primera crítica está dirigida hacia el diezmo, ya que alude a que viene de "tiempos remotos", es decir, una pesada herencia colonial. La segunda crítica está dirigida al Catastro agrícola, creada en 1831 para pagar los intereses del primer préstamo de Chile a Londres (Llorca-Jaña., et al, 2017). Al mismo tiempo, relaciona políticas económicas más liberales para la agricultura con la ciencia y filosofía, por lo que se subentiende una analogía entre los viejos impuestos, y las medidas de tendencia liberal como lo nuevo, lo que conduce a un progreso similar al de Inglaterra. En el mismo boletín, García Reyes hace referencia al artículo de agosto de 1839, que mencionamos anteriormente: "En una de aquellas memorias que corre impresa en El Agricultor, es debida seguramente la ley que hacia fines del año anterior, expidió la Legislatura por indicación del Gobierno, suprimiendo los derechos que pagaban los trigos a su salida del país" (El Agricultor, $\mathrm{N}^{\circ} 20$, diciembre de 1841: 7).

De esta manera, podemos observar como la SNA fue capaz de influir directamente en el Estado a través de sus importantes conexiones políticas. Un ejemplo de estas conexiones fue la constante participación de José Joaquín Prieto en las sesiones de la Sociedad mientras fue presidente de la República. Al momento de terminar sus funciones como mandatario, la Sociedad hizo una mención especial a modo de agradecimiento, denominándolo como "la columna que sostuvo el edificio vacilante" (El Agricultor, $\mathrm{N}^{\circ} 20$, diciembre de 1841: 18).

Otra fuerte crítica apareció en El Agricultor en 1848, en un artículo titulado "Necesidad que tiene la agricultura de una protección ilustrada, liberal y eficaz". El texto considera a los impuestos de la agricultura como injustos, que perjudican tanto al Estado como a los hacendados: "Cierto es que la multiplicada variedad de esta satisface directamente el doble fin que hemos indicado, de aumentar la riqueza pública y privada; pero cierto es también que mientras la una sea indispensable de la otra, todo abuso, lo mismo que toda desigualdad, debe comprometer la prosperidad de ambos" (El Agricultor, $\mathrm{N}^{\circ} 76$, julio de 1848: 494.). Por lo tanto, desde este punto de vista, desfavorecer a los hacendados con altos impuestos era también hacerlo con el Estado; es decir, un problema que afecta a los grandes propietarios se transforma en un problema inherentemente público.

En estricto rigor, el diezmo no fue eliminado, sino que fue reemplazado con otra contribución que tuviera los mismos objetivos, pero que fuera menos gravoso para los hacendados. En las sesiones del Congreso se puede observar una clara unanimidad al respecto. En este sentido, el diputado Matías Ovalle (miembro de la SNA, 
Ministro de Hacienda durante el año 1859 y con importantes conexiones con la agricultura ${ }^{4}$ ), aseguraba que "la conversión del diezmo en otro impuesto que no tenga sus inconvenientes, en la cual no hay disputa, porque todos convienen en su necesidad" (Cámara de Diputados. Sesión 25 ordinario en 8 de agosto de 1853: 166). El mismo Diputado comentó sobre la necesidad de eliminar el diezmo para competir con la creciente producción agrícola de California, ya que "para poner a nuestra agricultura en estado de lidiar con ventaja es necesario ponerla en aptitud de vender barato sus productos. Para conseguirlo se necesita desprenderla de los inconvenientes domésticos con que a la vez tiene que luchar, y que dan por resultado recargar sus frutos con costos inútiles a la producción" (Cámara de Diputados. Sesión 25 ordinario en 8 de agosto de 1853: 167).

También hubo un completo acuerdo en mantener el objetivo del diezmo: servir a los gastos de la Iglesia. Al momento de presentar el proyecto, el Presidente Manuel Montt (propietario de la hacienda Mercedes en la provincia de Santiago) y el Ministro de Hacienda, José Waddington ${ }^{5}$ (propietario de la hacienda San Pedro en la provincia de Valparaíso), se comunicaron con el Arzobispo de Santiago, Rafael Valentín Valdivieso (diputado en tres ocasiones y décimo propietario con mayores ingresos en los catastros de 1836-1837 y 1852), por medio de correspondencia, adjuntada en la Sesión en que se presentó el proyecto. En la carta, el arzobispo respondió al Ministro de Hacienda: "en nombre de la Santa Sede presto mi acuerdo para la aprobación del proyecto de conversión del diezmo en un impuesto directo a las propiedades [...] deben ser sin perjuicio de los derechos garantizados a la Iglesia" (Cámara de Diputados. Sesión 12 del 4 de julio de 1853: 74).

Para realizar la conversión del diezmo se establecieron dos propuestas: una desde el Gobierno y otra desde una Comisión compuesta por diputados. Ambas propuestas consideraban cambiar el $10 \%$ por un $5 \%$ de la contribución. El punto principal donde diferían ambas propuestas era si se impondrá respecto al valor de la propiedad (canon estable que ya estaba estudiado en el censo de 1852), o la otra, de acuerdo con el valor de la renta, es decir, al valor de la producción de cada fundo. La primera idea fue planteada por el Gobierno, mientras la segunda por la Comisión. Esta diferencia fue la que generó más debate y disputas en las dos Cámaras.

La concentración de los debates en este tema no era casualidad, dicha medida afectaba directamente los intereses de los hacendados. Bauer (1975) indicando la creación de un impuesto agrícola único en 1860, menciona que "se tributaba no por la propiedad en sí, sino por el ingreso agrícola (renta agrícola); así no se penalizaba la tierra ociosa" (144). Por ende, el proyecto de la Comisión tendía a perpetuar y facilitar la concentración de tierras ociosas. Matías Ovalle, defensor de lo planteado por la Comisión, explicaba el principal argumento de cobrar un impuesto acorde al valor de la producción: "ya que se trata de reformar el décimo, lo más acertado sería decrecer y distribuir mejor dicho tributo" (Cámara de Diputados. Sesión 22 ordinario en 1 de agosto de 1853: 148); es decir, que los impuestos se cobren de acuerdo a las ganancias que otorgue el fundo a su dueño, aludiendo a un argumento de equidad. Ramón 
Rengifo, criticando la propuesta de la Comisión, explicaba: "no sucedería de este modo si fuese a averiguarse al comerciante cuánto gana anualmente en su giro (...) Semejante procedimiento, a más de inicuo, sería impracticable" (Cámara de Diputados. Sesión 25 ordinario en 8 de agosto de 1853: 173).

En la Cámara de Senadores ocurrieron discusiones similares. En la primera discusión sobre el proyecto, Pedro Nolasco Mena (presidente la SNA desde 1839 a 1845. Diputado en siete ocasiones y una vez senador. Poseía una hacienda en Maipo) se mostraba totalmente en contra del proyecto presentado por el Gobierno, porque consideraba una grave desigualdad al momento de cobrar los impuestos. Ejemplifica, para ello, con dos haciendas de igual valor, pero donde una produzca mil, y la otra, cuatro mil fanegas de trigo. En dicho caso ambos deberían pagar la misma cantidad, significando que "no es proporcionalmente igual, porque carga igual al que ha cosechado mil que al que ha cosechado cuatro mil" (Cámara de Senadores. Sesión ordinaria en 5 de septiembre de 1853: 230). Como respuesta, el ministro del Interior, Antonio Varas sostuvo: "Sin duda, señor, este es el resultado del proyecto, y precisamente es lo que se quiere obtener". Desde el punto de vista de los partidarios del proyecto del Gobierno, pretenden que cobrar impuestos por valor de la propiedad sea un estímulo (aunque forzoso) para la agricultura. Por ese motivo, Varas responde a Mena: "El gravar la producción no es fomentarla, y yo prefiero el que se empleen grandes capitales en producir, a que esos mismos capitales se empleen en un suelo que no produzca nada" (Cámara de
Senadores. Sesión ordinaria en 5 de septiembre de 1853: 230).

Por su parte Mena contestó que "No se puede por otra parte obligar por fuerza a que los capitales se empleen en la agricultura; esto es obra del tiempo y de la conveniencia de los particulares". La sesión fue principalmente el debate entre Mena y Varas, solamente termina con la intervención del futuro presidente, José Joaquín Pérez: “por ahora me parece que la Cámara de Senadores no debe hacer otra cosa que aprobar en general el proyecto, reservándose para hacer en la discusión particular las observaciones que se crea convenientes" (Cámara de Senadores. Sesión ordinaria en 5 de septiembre de 1853: 234). A fin de cuentas, en 1854, la conversión del diezmo ya era efectiva, siendo aprobada la idea planteada por la Comisión.

La SNA, respecto a la conversión del diezmo, se presenta rotundamente a favor. Se hicieron pocas menciones al proyecto en El Mensajero, aunque fueron comentarios totalmente positivos, tachándolo de una "contribución mucho más equitativa y perceptible que la anterior" (El Mensajero I, 1856:54). De tal forma, consideraban definitivamente mejor el nuevo impuesto, ya que el antiguo gravamen era "un inútil embarazo, que sin aumentar las rentas fiscales, ponía un tributo al chileno activo y laborioso, alimentando la ociosidad y la pereza" (El Mensajero I, 1856: 138). Los detalles más precisos se presentaron en una Memoria del Intendente de Concepción, Rafael Sotomayor Baeza, que es publicada en El Mensajero. El Intendente comentó: “es un bien demasiado notable para que se deje de recordarlo 
en este lugar. Puede asegurarse que no hay un solo propietario, que por la nueva imposición pague una mitad de lo que adeudaría por diezmo" (El Mensajero II, 1857: 186).

\subsection{Crédito y banca}

Antes de 1855, los sistemas de crédito dependían esencialmente de casas comerciales, habilitadores o particulares que se encontraban en Santiago y, principalmente, en Valparaíso. Eran mecanismos informales y poco desarrollados, que tenían respaldo solamente en la palabra y reputación de los prestatarios. Según Eduardo Cavieres (1988), las principales realidades económicas del país durante las primeras décadas republicanas fueron "la inexistencia de un sistema bancario, la falta de inversiones productivas, el desconocimiento de técnicas de comercio moderno y prácticamente la ausencia de instrumentos de crédito" (117).

Por esta razón, desde el primer periodo de la SNA -El Agricultor- se expuso la necesidad de la instalación de un sistema formal de créditos para los hacendados. Demanda que se materializó en 1855 con la creación de un mecanismo de crédito estatal: la Caja de Crédito Hipotecario. Esta institución fue "un tipo de banco hipotecario agrícola organizado para para otorgar préstamos a largo plazo a los agricultores, bajogarantía hipotecaria de sus propiedades" (Cavieres, 1988. 136). Desde 1855, se crearon varios bancos en el país, pero solamente la Caja es creada desde el Estado. Los demás fueron iniciativas de privados, a través de Sociedades Anónimas.
Primeramente, la preocupación de la SNA fue la creación de una Caja de Ahorros, el primer artículo en proponer una institución de este tipo se encuentra en agosto de 1841, planteando dos objetivos principales: “1) que inspire la más decidida confianza en los pequeños capitalistas que quieran entregarle sus ahorros, presentándoles garantías cuantiosas y exentas de toda tacha. 2) Combinar los intereses de todos los que tengan parte en él; de modo que solo esta combinación le de consistencia necesaria, sin recurrir a apoyos extraños" (El Agricultor, $\mathrm{N}^{\circ}$ 18, agosto de 1841: 76). Como se puede observar, la Caja es una institución donde se pueden depositar los capitales para su mejor administración, también la proponen como una iniciativa de privados, en la cual, personas con grandes riquezas aportan la mayor parte del capital de la Caja. La propuesta es aprobada por el gobierno un año después, en agosto de 1842 . Uno de los objetivos enunciado en numerosas ocasiones por la Sociedad respecto a los bancosy la Caja de ahorros, es que, según ellos, tiene una capacidad moralizadora, fomentando el trabajo y erradicando los vicios, tanto en las clases populares como de las acomodadas. En 1846 Wenceslao Vial propone un proyecto (posteriormente aprobado) para inscribir a los "artesanos milicianos" en la Caja de Ahorros, con el objetivo de "al mismo tiempo, el mejor servicio de la guardia nacional, la moralidad de los artesanos y su mejora física" (El Agricultor, $\mathrm{N}^{\circ}$ 57, mayo de 1846: 2).

Las limitadas funciones de la Caja de Ahorros, generaron que, en 1844, el problema por la obtención de créditos se hiciera presente con gran fuerza en la SNA. En octubre de 1844 publicaron un largo artículo, de 123 páginas, que lleva como 
título principal: "Proyecto de Banco", escrito por Mariano Fragueiro6. En las últimas páginas de la publicación, la Sociedad afirmó que "acordó después de largas y maduras discusiones: que era conveniente en Chile el establecimiento de un Banco", de esta manera "Después del examen que hemos indicado pasó la Sociedad a considerar el proyecto del sr. Fragueiro: y aprobó sus bases y términos" (El Agricultor, ${ }^{\circ} 50$, octubre de 1844: 122).

En un primer momento, se exponen una serie de artículos que Fragueiro había publicado en el diario El Progreso; en ellos, explica, principalmente, cuestiones técnicas: formación de bancos, de los giros y depósitos, convertibilidad de los valores de un banco, fiscalización de los billetes, etc. Las ideas más importantes del autor se relacionan a la gestión y origen del banco, que a su juicio "deben ser administrados por un cuarto poder político independiente, como los tres ya conocidos en los Gobiernos constitucionales [...] Cuando la ley haya creado una administración de crédito independiente del ejecutivo, el crédito no será otra cosa que el movimiento de los valores por entre las manos de administradores inteligentes y próvidos, responsable de su conducta ante la ley y garantidos en todo caso por el Estado"( El Agricultor, $\mathrm{N}^{\circ} 50$, octubre de 1844: 2-3). También se expuso una carta enviada a Ramón Luis Irarrázaval, ministro del Interior y Relaciones Exteriores (quien también era socio de la SNA y hermano José Miguel Irarrázaval, el décimo primer propietario de mayores ingresos para los años 1837-1837), pidiéndole que evalúe la implementación de un banco en Chile, bajo las consideraciones que él ha expuesto. La respuesta de Irarrázaval es cordial y positiva, afirmando que "Yo señor, no encuentro otra cosa que objetar a su luminosa Memoria" y que por lo tanto presentará estas ideas al presidente de la República (El Agricultor, $\mathrm{N}^{\circ}$ 50, octubre de 1844: 93).

Dentro del mismo Número se publica un “Oficio que la Sociedad de Agricultura eleva al Supremo Gobierno", redactado por Pedro Félix Vicuña. El objetivo del Oficio es mostrar al Gobierno la necesidad de una institución que entregue créditos a las industrias nacionales: "La escasez de capitales para impulsar la industria, que parece despertarse, es el escollo en que han fracasado nuestras esperanzas y buenos deseos" (El Agricultor, $\mathrm{N}^{\circ} 50$, octubre de 1844: 100). Por lo tanto, desde la SNA piensan que el poco desarrollo de las actividades económicas chilenas se debe a la falta de capitales que imposibilita la inversión de los empresarios y agricultores en sus respectivas actividades. Como es sabido, dichas ideas no se concretan; la creación de un banco durante la década de 1850 estuvo a cargo de mineros y comerciantes (extranjeros y nacionales), que se organizaron en Sociedades Anónimas. Solamente la Caja de Crédito Hipotecario fue una institución estatal con funciones similares a las de un banco.

La preocupación por la obtención de crédito se hizo presente nuevamente en 1848, en un extenso artículo que enumeró las reformas necesarias para el mejoramiento de la agricultura: eliminación de mayorazgos, fomento de la inmigración europea, policía rural, mejorar las condiciones de vida de los campesinos, y la creación de Banco de fomento de la agricultura. Respecto al último punto, consideraban que era importante, ya que 
los escasos de capitales "embarazan toda empresa industrial" y la Caja de Ahorros no es suficiente para dicha tarea: "por más que las consideremos en toda la extensión de sus benéficos resultados, nos esfuerza reconocer que su influencia es más limitada que la de un banco" (El Agricultor, $\mathrm{N}^{\circ}$ 76 , julio de 1848: 526.). Un banco de fomento es diferente a un banco corriente, ya que no posee tantas atribuciones, por ende, sus funciones son más parecidas a las de la futura Caja de Crédito Hipotecario: "La vasta teoría de los establecimientos de crédito no es aplicable a los bancos de fomento, porque la influencia de estos aunque extensa, no es mayor que la de una empresa particular" (El Agricultor, $\mathrm{N}^{\circ} 76$, julio de 1848: 527), es decir, sus tareas son principalmente la entrega créditos a los agricultores.

Sin embargo, a diferencia de la Caja de Crédito Hipotecario, el banco de fomento fue pensado como una institución privada, similar a una Sociedad Anónima, porque de ella "se reducen a asociaciones de capitalistas, que por medio de acciones reúnen un fondo para hacer cortos adelantos a los pequeños propietarios [...] recibiendo en garantía de las obligaciones del deudor la hipoteca de sus propiedades" (El Agricultor, $\mathrm{N}^{\circ}$ 76 , julio de 1848: 528). Como es posible observar, la idea de este banco de fomento es beneficiar a los pequeños agricultores, iniciativa que durante la creación de la Caja Crédito Hipotecario fue totalmente olvidada.

En resumen, desde El Agricultor se presentó una preocupación por la obtención de créditos. En un primer momento y con menos inquietud sobre el tema, se creó una Caja de Ahorros, de limitadas funciones, pero que de igual manera apuntaba a suplir la carencia de instituciones bancarias. Ya en 1844, la necesidad de un banco era notoria: la Sociedad dejó de publicar desde octubre de 1844 hasta noviembre de 1845 , demostrando su preocupación por el "Proyecto de Bancos" de Mariano Fragueiro. El proyecto, tuvo la función de poner en debate las cuestiones técnicas de los bancos, por lo tanto, existe la intención de informar a los socios en temas bancarios y de crédito. En 1848, se planteó la idea de crear un banco de fomento, ya que el problema identificado por la Sociedad es claro: la falta de bancos o instituciones de crédito no permite que las actividades agrícolas se desarrollen de una manera competente.

De esta manera, en junio de 1855 el Gobierno envió a las Cámaras legislativas el proyecto para establecer la Caja de Crédito Hipotecario, texto redactado por el ministro del Interior Antonio Varas. En la presentación del documento, se destaca que "El autor del proyecto del banco de fomento a la agricultura ha querido favorecer el más importante ramo de industria del país", ya que lo que más necesita la agricultura son "recursos fijos, estables con que siempre pueda contar" (Cámara de Senadores. Sesión 3ra, en 11 de junio de 1855: 15). La formación de la Caja fue parte fundamental del programa que ha mantenido el Gobierno de Montt. En el Manifiesto del Partido Conservador (1851) se afirma: "Chile es una nación esencialmente agrícola y comercial. De modo que todo lo que contribuya a dar vuelo a estas industrias, todo lo que haga desaparecer los obstáculos que los países nuevos obstan a su desarrollo, debe ser objeto de las bendiciones del pueblo". Incluso, llaman a la agricultura "nuestra 
principal industria”. Es importante mencionar que en el Manifiesto se presenta la lista de electores para presidente de la República, de los 20 diferentes puestos, proponen a 12 personas que fueron socios de la SNA durante su primer periodo.

Los debates en ambas Cámaras avanzaron rápidamente. Respecto a los senadores, la discusión más larga fue entre Arístegui, que proponía excepciones de penalización para los eclesiásticos -haciendo valer el fuero-y el senador Mujica, quien rebatía esa idea. La propuesta de Arístegui fue rechaza por 10 votos contra 3. En la Cámara de Diputados el proyecto también avanzó rápidamente. No obstante, es importante mencionar los aportes del diputado y futuro presidente de la República Federico Errázuriz, miembro de la SNA y propietario en Colchagua con una hacienda evaluada en 9.000 pesos (Bauer, 1975: 48). Errázuriz propuso realizar importantes cambios al artículo 16 , específicamente a extender a: "dos meses el plazo que se conceden al deudor para el pago de lo que adeudare". La segunda propuesta era eliminar la facultad de la Caja "que le habilita para pedir y tomar la posesión de fundo hipotecado”. Errázuriz argumenta: "Como sea que se mire, es sin disputa que despojar de la posesión al propietario, no es otra cosa que labrarle su completa ruina" (Cámara de Diputados. Extracto de la sesión 12, en 14 de julio de 1855: 64-65).

En cuanto a la primera idea de Errázuriz, se votó a favor de una enmienda que el ministro Varas hizo al diputado. Mientras que su segunda indicación fue desechada por 36 votos contra 4. Errázuriz estuvo, claramente, en favor de los intereses de los hacendados: en la sesión del 19 de julio se procedió a "continuar la discusión particular del proyecto que reglamenta los derechos y obligaciones reciprocas de los maestros y oficiales de talleres", propuesta iniciada por Francisco Ramírez, y que no tiene relación con los debates sobre la Caja de Crédito. Por ese motivo, "el señor Errázuriz, pidió que se difiriese la consideración de este asunto, hasta después que la Cámara se hubiese pronunciado sobre otros proyectos ante ella pendientes, y de más urgencia e importancia que el puesto en discusión; tales como el concerniente al establecimiento de un Banco Hipotecario". La declaración de Errázuriz disgustó a Ramírez, ya que "Tres años ha que fue presentado el proyecto, y solo ahora se llega a su discusión particular; y no obstante se pide su postergación sin objeto fundamental" (Cámara de Diputados. Extracto de la sesión 5, en 19 de junio de 1855: 46). La petición de Errázuriz es aprobada por 27 votos contra 11. Así, a causa de la necesidad de los hacendados, y el acuerdo general en la elite chilena en establecer una institución de este tipo, provocó que el proyecto avanzara ágilmente por ambas Cámara legislativas. En 1856 la Caja comienza sus funciones.

La SNA, desde El Mensajero, consideró la creación de la Caja de Crédito Hipotecario como una institución de indiscutido valor, que "tal cómo está organizada, se halla en cierto modo corroborada por las circunstancias actuales del país" (El Mensajero I, 1856: 158). De acuerdo a un texto publicado en El Mensajero, "El Banco Hipotecario, sancionado por la última legislatura, será la institución más protectora de los intereses de la agricultura en el sud: con la facilidad que él presta a los agricultores para procurarse de capitales a bajo interés, lo 
alivia de la pesada carga que sufre" (El Mensajero II, 1857: 185).

La Caja de Crédito Hipotecario fue el resultado de las exigencias y presiones de la elite agraria para obtener una fuente segura de créditos. La expansión de la agricultura a causa del ciclo triguero californiano fue el momento ideal para establecerla, de este modo "la creación de ella ha sido sugerida por una bien entendida reflexión sobre la industria que requiera más fomento, empuje y protección en nuestro país" (El Mensajero I, 1856: 158). Por ello, Borde y Góngora (1956) la describen como un dócil instrumento en manos de los terratenientes. Sin embargo, desde la Sociedad hacen algunas críticas a determinados aspectos. Uno de ellos tiene relación con la exclusión de los pequeños propietarios de la participación de sus beneficios, ya que "conviene solo a los grandes propietarios, y por su forma y estatutos muy poco accesibles a los demás que son quizás los que más necesitan del crédito y en los que mejor efecto surtiera" (El Mensajero I, 1856: 157).

Otro problema que identifican en la SNA, son las dificultades que tienen los hacendados que viven lejos de Santiago para realizar trámites relacionados con la Caja: "La organización de la Caja pueden ser muy buena y entendida, y el no dar todos los resultados apetecibles no provienen de ella misma sino de la poca extensión que tiene para obrar y el no ser fácilmente abordable a todos los agricultores que se hallan distantes de ella" (El Mensajero I, 1856: 159). Si bien hubo críticas desde la Sociedad a la Caja, no fueron relevantes, a rasgos generales la institución pudo servir con efectividad a los intereses de los grandes propietarios y, por lo tanto, fue muy bien recibida. Pero no pudo lograr cambiar las actitudes rentistas de los terratenientes, es decir, no tuvo efecto en "fomentar el espíritu de ahorro, economía y arreglo" (El Mensajero I, 1856: 158). Por el contrario, fomentó el consumo conspicuo y la consolidación del poder de los hacendados. En 1857, Vicuña Mackenna, poco antes de renunciar a su puesto en la SNA, comentó: "El Gobierno ha establecido un banco hipotecario para impulsar los intereses rurales. ¿Quién ha invertido alguna suma en provecho de sus campos? ¡Respondan por nosotros los palacios de la Capital!" (El Mensajero II, 1857: 254).

Se podría argumentar que la creación de la Caja de Crédito Hipotecario fue motivada, principalmente, por la prosperidad que experimentaba la agricultura. Sin embargo, la minería durante esa misma época también estaba aumentando considerablemente su producción. Entre 1851 y 1861 Chile desplazó a Inglaterra como el mayor productor de cobre, quedando en primer lugar, con un $32 \%$ de la producción mundial, mientras que Inglaterra con un $21 \%$ (Nazer, 1994). Por lo tanto, la creación de una institución de crédito estatal para los terratenientes, no se debe únicamente a la prosperidad de la agricultura, sino también, a la gran influencia de la elite agraria en el Estado.

\section{Conclusiones}

El sector agrario estuvo vinculado desde el comienzo de la independencia a los más importantes puestos estatales. Esta condición de predominio en el Estado les posibilitó influir en las principales esferas de la política nacional. Los estudios sobre 
los terratenientes chilenos durante el siglo XIX se han concentrado en las décadas posteriores a 1860, cuando comenzaron las exportaciones a Inglaterra. Por otro lado, los análisis son cuantitativos, destacando su presencia en el Congreso y los gobiernos. Por ende, previo a 1860, el sector más influyente de la sociedad chilena carecía de una profundización en cuanto a su práctica política.

En este sentido, nuestra investigación, permite un acercamiento a la relación entre el Estado y la elite agraria a mediados del siglo XIX. Evidenciando, como los intereses de la elite agraria, se materializaron en políticas que benefician directamente a los hacendados. Analizando dos temas fundamentales para el sector agrario, impuestos y crédito, constatamos que la elite agraria tuvo importantes instancias de participación política. Una de ellas fue la Sociedad Nacional de Agricultura, una institución que permitió agrupar a un importante número de la elite nacional, en torno a los intereses agrarios. Por medio de la Sociedad, lograron promover ideas y planteamientos, pero también funcionó como un puente entre la elite agraria y el Gobierno.

Utilizamos la creación de la Caja de Crédito Hipotecario y la conversión del diezmo, como la materialización de sus influencias, pero, además, como un punto de referencia para realizar nuestro análisis. De esta manera, previo a la promulgación de dichas leyes, por medio de El Agricultor, comprendemos las inquietudes e ideas del sector agrario. Luego, a través de las sesiones del Congreso nacional, evidenciamos la presencia de actores vinculados a la agricultura que transitan entre el parlamento, el gobierno y la SNA. Desde esos espacios, dichos actores defienden los intereses agrícolas e impulsan leyes en su favor. Por último, en publicaciones de El Mensajero, podemos conocer las opiniones de la elite agraria después de la promulgación de ambas leyes. Opiniones que claramente muestran conformidad y simpatía por las medidas tomas por el gobierno de Manuel Montt.

El origen del propósito de esta investigación era comprender cómo funcionaba y qué factores influían en la toma de decisiones políticas durante los primeros gobiernos de la República, con el objetivo de comprender los primeros pasos de la organización nacional y estatal. Ello, tuvo como resultado enfocar el análisis hacia quienes tenían mayor influencia en la sociedad chilena, los terratenientes. No obstante, debido al alcance del presente artículo, quedan preguntas que condicen con nuestra investigación. Una de ellas, la que creemos más importante, sería comprender la visión y opinión de la elite agraria sobre pequeños campesinos e inquilinos. Afortunadamente, las dos primeras revistas de la SNA, tienen bastante información sobre este tema, especialmente algunos boletines de El Agricultor. Otro tema que puede proseguir de esta investigación, es la influencia de los intereses agrarios a nivel municipal, pero como es sabido, la principal dificultad para ello, es la escasa cantidad de fuentes durante la primera mitad del siglo XIX.

\section{Referencias citadas}

Acosta, A. (2014): Los orígenes de la burguesía de El Salvador. El control sobre el café y el Estado. 1848-1890. Sevilla: Taller de estudios e investigaciones Andi- 
no-Amazónicos.

Bauer, A. (1975): La sociedad rural chilena, desde la conquista española a nuestros días. Santiago: Editorial Universitaria.

Bengoa, J. (2015): Historia rural de Chile central. Tomo I: la Construcción del Valle Central de Chile. Santiago: LOM Ediciones.

Borde, J. y M. Góngora (1956): Evolución de la propiedad rural en el Valle del Puangue. Tomo I. Santiago: Editorial Universitaria.

Carmagnani, M. (1984): Estado y Sociedad en América Latina (1850-1930). Barcelona: Ed. Hurope.

Cavieres, E. (1988): Comercio chileno y comerciantes ingleses. 1820-1880. Santiago: Editorial Universitaria.

Izquierdo, G. (1968): Un estudio de las ideologías chilenas. Santiago: Imprenta Técnica LTDA

Joignant, A. (2009): El estudio de las Élites. Un estado del arte. Santiago: Universidad Diego Portales.

Llorca-Jaña M., Claudio R., Javier N. y Roberto A. (2017): "La agricultura y la elite agraria chilena a través de los catastros agrícolas, c.1830-1855”, Historia, 50, pp. 597-639.

Mattelart A., Carmen C. y Leonardo C. (1970): La ideología de la dominación en una sociedad dependiente. Santiago: Ediciones Signos.

Nazer, R. (1994): José Tomás Urmeneta. Un empresario del siglo XIX. Santiago: Dirección de Bibliotecas,
Archivos y Museos.

Robles, C. (2003): “Expansión y transformación de la agricultura en una economía exportadora. La transición del capitalismo agrario en Chile (1850-1930)", Historia Agraria, 29, pp. 45-80.

Robles, C. (2007): Hacendados progresistas y modernización agraria en Chile Central (1850-1880). Osorno: Universidad de los Lagos.

Sagredo, R. (1996): "Élites chilenas del siglo XIX. Historiografía", Cuadernos de Historia, 16, pp. 103-132.

Sepúlveda, S. (1959): El trigo chileno en el mercado mundial: ensayo de geografía histórica. Santiago: Editorial Universitaria.

Véliz, C. (1961): Historia de la Marina Mercante de Chile. Santiago: Ediciones de la Universidad de Chile.

\section{Fuentes}

Sociedad Nacional de Agricultura:

-El Agricultor. 73 números de 78. Faltan los n 55, 56, 64,68 у 77.

-El Mensajero de la Agricultura I 1856 y II 1857.

-Sesiones de los Cuerpos Legislativos. 1851 a 1855.

- Manifiesto del Partido Conservador a la Nación. Folleto publicado en mayo de 1851. 


\section{Notas}

1El concepto es utilizado por Llorca-Jaña et. al. (2017), aunque los autores no especifican la misma definición que hemos descrito en el presente artículo.

${ }^{2}$ Denominaremos a éstas demandas de California y Australia como "ciclo triguero californiano", ya que ambas demandas serán consideradas en conjunto.

${ }^{3}$ Concepto ocupado por Robles (2007).

${ }^{4}$ Sus tíos maternos figuran entre los diez agricultores más acaudalados en la década de 1830: Isidoro, Fernando y Ramón Errázuriz Aldunate; igualmente su hermanastro Francisco Javier Ovalle Errázuriz fue dueño de una hacienda en Marchigüe; mientras que su padre Ramón Ovalle de Vivar fue propietario de una hacienda ubicada en La Calera.

${ }^{5}$ Hijo de Josué Waddington, un comerciante británico, y hermano de Joshua Waddington, quien fue un poderoso comerciante que diversificó sus inversiones en otros sectores, incluyendo el agrario, para el año 1852 figura en el número 38 de los propietarios con mayores ingresos.

${ }^{6}$ Fue un importante político y financista argentino. Tuvo una larga carrera política en su país, y además fue parte del directorio del Banco de Buenos Aires. En Chile realizó negocios relacionados con la minería y fue autor del proyecto del ferrocarril Caldera-Copiapó. 